\title{
Der affektive Einfluss von politischer Werbung in Kampagnen auf Einstellungen
}

\section{Christian Schemer}

Die Studie analysiert den Einfluss von politischer Werbung auf die Einstellungen von Rezipienten im Verlauf der Kampagne zur Asylgesetzabstimmung in der Schweiz 2006. Auf Basis von Daten aus einer dreiwelligen Panelbefragung wird insbesondere der Einfluss von politischer Werbung auf die Einstellung untersucht, der durch positive und negative Emotionen vermittelt wird. Es zeigt sich, dass insbesondere die Befürworter der Asylgesetzverschärfung durch ibre Kampagnenwerbung negative Emotionen bei den Befragten auslösten (z. B. Angst und Ärger gegenüber Asylsuchenden) und dadurch die Unterstützung für eine Verschärfung des Asylgesetzes erböhen konnten. Positive Emotionen, wie Freude oder Hoffnung, wurden durch die politische Werbung nicht erregt. Damit zeigt die Studie, dass affektive Wirkungen, die bisher nur im Experimentallabor nachgewiesen wurden, auch im Verlauf realer Kampagnen unter zweiseitigen Kommunikationsbedingungen auftreten können.

Schlagwörter: Politische Werbewirkung, Emotionen, Einstellungen, latentes Wachstumskurvenmodell

\section{Einleitung}

Es ist sicher unbestritten, dass politische Akteure in Kampagnen nicht nur den Verstand, sondern auch das „Herz“ von Wählerinnen und Wählern ansprechen wollen (vgl. bereits Hartmann 1936; Lazarsfeld et al. 1944; vgl. auch Dahlem 2001). Zu der Frage, ob solche Kampagnenstrategien die Emotionen von Wählerinnen und Wählern tatsächlich erregen, liegen jedoch kaum empirische Studien unter extern validen Bedingungen vor. Darüber hinaus fehlen Erkenntnisse darüber, ob diese in Kampagnen aktivierten Emotionen einen Einfluss auf die Einstellung oder die Wahlabsicht haben. Eine Vielzahl von Experimenten zeigt zwar, dass beispielsweise Wahlwerbespots Emotionen bei Rezipienten auslösen (z. B. Brader 2006; Kaid 2004; Thorson et al. 1991). Allerdings sind diese Befunde nicht ohne Weiteres generalisierbar, weil Rezipienten im Experiment einer einseitigen und kurzzeitigen Kommunikation ausgesetzt sind. Darüber hinaus ist unklar, wie lange solche Wirkungen andauern können, zumal die Halbwertszeit von Emotionswirkungen, die von diesem einseitigen Stimulusmaterial ausgeht, kaum länger als ein paar Tage ist (vgl. hierzu Schemer 2009a: 106ff.).

Neben experimentellen Untersuchungen liegen zwar auch Befragungsstudien zum Einfluss von Emotionen auf politische Einstellungen vor, z. B. gegenüber politischen Kandidaten (Abelson et al. 1982; Marcus/MacKuen 1993; Schoen 2006). Diese Studien sind aber aus zwei Gründen eingeschränkt: erstens aufgrund der Datenlage, weil es sich in der Regel um Querschnittdaten handelt. Wenn in diesen Studien Emotionseinflüsse auf Einstellungen nachgewiesen werden, dann basiert diese Schlussfolgerung auf einer Korrelation von Emotionen und der Einstellung. Statt aber aus dieser Korrelation zu folgern, dass Emotionen die Einstellung beeinflussen, könnte ebenso gut angenommen werden, dass eine bestimmte Einstellung eine Emotion auslöst oder aber dass sowohl die Einstellung als auch die Emotion durch eine dritte Variable verursacht werden. Zweitens sind die vorliegenden Befragungsstudien eingeschränkt, weil sie nicht untersuchen, woher eigentlich die Emotionen stammen, die sich auf die politische Einstellung auswirken. 
Mit anderen Worten, es wird nicht untersucht, ob Emotionen tatsächlich durch Kampagnenkommunikation, z. B. politische Werbung, verursacht wurden oder ob es sich um affektive Prädispositionen handelt, die überhaupt nicht durch die Kampagne ausgelöst wurden, sondern stabile affektive Reaktionen gegenüber politischen Kandidaten oder Sachverhalten darstellen, die im Laufe der politischen Sozialisation erworben wurden.

Daher widmet sich die vorliegende Studie der Frage, wie durch politische Werbung induzierte Affekte die Einstellung von Rezipienten im Verlauf einer realen politischen Kampagne beeinflussen. Der Beitrag ist wie folgt gegliedert: Zunächst wird das vorliegende Verständnis von Emotionen bzw. Affekten dargelegt. Anschließend folgt ein Überblick über a) inhaltsanalytische Studien, die zeigen, dass die politische Kommunikation in Kampagnen darauf angelegt ist, Emotionen in der Wählerschaft auszulösen, und b) experimentelle Forschung, die nachweist, dass bestimmte Medienangebote affektive Reaktionen kausal verursachen. Schließlich folgt eine kurze Darstellung von Befragungsstudien zu den affektiven Einflüssen auf politische Einstellungen in Kampagnen. Auf Basis dieser bisherigen Studien werden die Forschungsfragen für die folgende Untersuchung abgeleitet und im Methodenpart das Vorgehen der Untersuchung deutlich gemacht. Auf die Ergebnisse folgt ferner eine eingehende Diskussion der Resultate vor dem Hintergrund der bisherigen Forschung.

\section{Das Verständnis von affektiven Einflüssen auf politische Einstellungen}

In der bisherigen Forschung zum Affekteinfluss auf politische Einstellungen wird Affekt als Überbegriff für jegliche Emotionen, Stimmungen oder Gefühle aufgefasst, die sich nur hinsichtlich ihrer Valenz unterscheiden (vgl. hierzu auch Otto et al. 2000). Beispielsweise werden in der Pionierstudie von Abelson et al. (1982) die Befragten gebeten anzugeben, ob sie jemals eine bestimmte emotionale Reaktion, z. B. Angst, Ärger oder Hoffnung gegenüber einem politischen Kandidaten, z. B. Ronald Reagan, verspürt haben. Die Antworten zu diesen selbstberichteten Emotionen ließen sich faktorenanalytisch zu positiven und negativen Affekten verdichten, durch die dann die Kandidatenpräferenz vorhergesagt werden konnte (vgl. auch Schmidt-Atzert 2000). ${ }^{1}$

Im Unterschied zu Affekten sind Emotionen sehr spezifisch und lassen sich nicht nur hinsichtlich ihrer Valenz unterscheiden, sondern auch anhand weiterer Bewertungsaspekte. In appraisaltheoretischer Lesart sind Emotionen spezifische Reaktionen auf die kognitive Bewertung von Umweltsituationen (vgl. als Überblick Ellsworth/Scherer 2003; Scherer et al. 2001). So stellen beispielsweise Angst und Ärger zwar negative Affekte dar, unterscheiden sich aber erheblich in ihren Ursachen bzw. Folgen für das Individuum. Spezifische Emotionen sind abgesehen von psychischen Erkrankungen jedoch eher von kurzer Dauer. Schließlich sind Affekte noch von Stimmungen abzugrenzen. Stimmungen sind im Unterschied zu Emotionen und Affekten nicht objektbezogen, sondern stellen eine Art affektiven Hintergrund für Individuen dar, der sich situativ auf die Wahrnehmung und Beurteilung von Objekten auswirken kann. Zusammenfassend wird also unter Affekten oder affektiven Reaktionen ein Syndrom spezifischer Emotionsreaktionen gegenüber einem politischen Einstellungsobjekt auf einem höheren Abstraktionsniveau als Emotionen verstanden. Affekte lassen sich lediglich noch im Hinblick auf ihre Valenz im Sinne von positiv und negativ unterscheiden. Im Unterschied zu Emotionen sind sie weniger spezifisch, im Unterschied zu Stimmungen jedoch ob-

1 Zum Teil findet sich auch in der Forschung ein Verständnis von Affekt, das synonym zur Einstellung gegenüber einem Objekt ist (z. B. Schimmack/Crites 2005). Ein solches Verständnis wird hier - wie auch im überwiegenden Teil der Forschung - aber nicht geteilt. 
jektbezogen. Wenn von affektiven Kampagnen- oder Medienwirkungen gesprochen wird, dann ist damit der Affekte auslösende Einfluss der Medien beim Rezipienten gemeint. Wenn von affektiven Einstellungswirkungen der Kampagne oder der Medienberichterstattung die Rede ist, dann sind damit die Medien- oder Kampagnenwirkungen auf Einstellungen gemeint, die durch Affekte mediiert werden. Das bedeutet die Kampagne löst Affekte aus, die dann die Einstellung beeinflussen.

Die vorliegende Studie geht dabei von einer strukturellen Perspektive auf Einstellungen aus. Demnach stellen Einstellungen abstrakte Konstrukte dar, die durch Komponenten auf niedrigerem Abstraktionsniveau beeinflusst werden. Eine solche Modellvorstellung ist etwa das Dreikomponentenmodell der Einstellung, das auf Rosenberg und Hovland (1960) zurückgeht. Dieses postuliert, dass Einstellungen Globalpräferenzen darstellen, die durch die Komponenten Kognitionen, Affekte und das Verhalten beeinflusst werden (vgl. hierzu allgemein Witte 1992, Zanna/Rempel 1988). Kognitionen, Affekte und Verhalten stellen demnach distinkte Konstrukte dar, die sich auch von der Einstellung als Globalpräferenz auf einem höheren Abstraktionsniveau unterscheiden lassen (z. B. Breckler 1984). Dieser Sichtweise folgt auch die vorliegende Modellannahme. Sie geht davon aus, dass Individuen eine bestimmte Einstellung zu einem $\mathrm{Ob}$ jekt haben können, die mit affektiven Reaktionen zu dem Objekt zusammenhängt. Weiter wird angenommen, dass Affekte zu einem Einstellungsobjekt und die Einstellung dazu distinkte Konstrukte im Sinne der Konstruktvalidität darstellen.

\section{Forschungsüberblick}

\subsection{Inhaltsanalysen zu Affektappellen und Emotionalisierung in der politischen Kommunikation}

Die politische Berichterstattung oder politische Werbespots zeichnen sich durch inhaltliche oder formale Merkmale aus, die mit großer Wahrscheinlichkeit affektive Reaktionen beim Publikum verursachen (z. B. Brader 2006; Donsbach/Büttner 2005; Kaid/ Holtz-Bacha 1995, 2006). Diese Botschaften können als Affektappelle bezeichnet werden. Beispielsweise zeigt eine Inhaltsanalyse von Werbespots in US-Wahlkämpfen, dass 84 \% dieser Spots Affektappelle als verbale Komponenten enthalten. Bei 44 \% der Werbespots ist der Affektappell sogar der dominante Aspekt des Spots (Kaid/Johnston 2001: $55)$.

Im deutschsprachigen Raum wurde darüber hinaus nachgewiesen, dass auch das Nachrichtenangebot Merkmale enthält, die geeignet sind, Emotionen im Publikum auszulösen (Donsbach/Büttner 2005; Krüger/Zapf-Schramm 2003; Ruhrmann et al. 2003). Dabei kann es sich um inhaltliche Merkmale handeln, wie etwa die Themenwahl (z. B. Kriminalität, Katastrophen), das Zeigen von Emotionen von Akteuren (z. B. ein weinendes Kind, verärgerte Politiker), die Verwendung einer lebhaften, metaphorischen Sprache sowie von Reizwörtern und werthaltigen Begriffen. Es kann sich aber auch um formale Aspekte der Berichterstattung handeln, wie etwa die Art der Visualisierung, Dynamik von Beiträgen (z. B. Schnitte oder Kameraführung) oder die Musikuntermalung von Nachrichtenbeiträgen, die Emotionen auslösen. Warum ausgerechnet solche Merkmale als Affektappelle oder Aspekte von Emotionalisierung angesehen werden, zeigt sich letztlich in experimentellen Untersuchungen, die den Kausalnachweis erbringen, dass bestimmte Merkmale des Medienangebots tatsächlich affektive Reaktionen verursachen. 


\subsection{Affektive Wirkungen der politischen Kommunikation in Kampagnen}

Die inhaltlichen Merkmale, die als Emotionalisierungsaspekte in Medienbeiträgen aufgefasst werden können, werden in der Regel aus den Ergebnissen experimenteller Untersuchungen abgeleitet. Beispielsweise zeigt sich in experimentellen Studien, dass die Kontextualisierung von Nachrichten im Zusammenhang mit bestimmten Themen, wie Krieg oder Kriminalität, Emotionen bei Rezipienten auslösen. Igartua et al. (2007) belegen, dass ein Medienbeitrag über Immigranten im Zusammenhang mit Kriminalität im Unterschied zu den volkswirtschaftlichen Vorteilen der Immigration vermehrt zu negativen affektiven Reaktionen, wie Angst, und weniger zu positiven affektiven Reaktionen, z. B. Freude, führte. Ein solcher Beitrag löste hingegen nicht nur vermehrt negative affektive Reaktionen aus, sondern hatte auch entsprechende Folgen für die Einstellung. Diejenigen Versuchspersonen, die den Beitrag im Zusammenhang mit Kriminalität anschauten, hatten auch eine affektkongruente - und damit negative bzw. restriktive Einstellung gegenüber der spanischen Immigrationspolitik. Bei den Probanden, die den Beitrag im Zusammenhang mit dem volkswirtschaftlichen Nutzen sahen, traten nicht nur positivere Emotionen auf, sondern auch eine positivere Einstellung zur Immigrationspolitik (vgl. für ähnliche Befunde auch Scheufele/Gasteiger 2007).

Die Forschung zur Wirkung von Kampagnenwerbespots hat ebenfalls Merkmale extrahiert, die affektive Reaktionen in der Wählerschaft auszulösen vermögen, z. B. Musikunterlegung, Verwendung von Symbolen wie Nationalflaggen, Schlüsselreize, wie Kindchenschema usw. (vgl. hierzu auch Brader 2006). Beispielsweise zeigen Thorson et al. (1991), dass personalisierte Wahlwerbespots weniger positive Emotionen erregen als sachorientierte bzw. argumenthaltige Spots. Ebenso weisen sie nach, dass Angriffe auf den politischen Gegner positive Emotionen beim Publikum dämpfen, während Unterstützungswerbung positive Emotionen verstärkt. Die Autoren belegen zwar auch Werbewirkungen auf die Einstellung gegenüber dem politischen Kandidaten, die das Ergebnis der affektiven Reaktionen sein könnten. Sie bleiben aber den empirischen Nachweis schuldig, dass die durch die Werbung verursachten Emotionen tatsächlich die Einstellung beeinflusst haben. $\mathrm{Zu}$ ähnlichen Ergebnissen kommen auch Brader (2005) sowie Kaid und Holtz-Bacha (1993) in der einzigen Studie zu affektiven Einflüssen von Wahlwerbung in Deutschland. Letzere wiesen nach, dass affektive Reaktionen nach dem Betrachten von Wahlwerbung für Helmut Kohl mit der Einstellung zum Kandidaten korrelierten (vgl. zum Einfluss politischer Werbung allgemein auch Maier/Maier 2007). Auch hier bleibt aber unklar, ob die Werbung die Emotionen verursacht, die dann die Einstellung prägen. ${ }^{2}$

Die bislang einzige Studie, die die Kausalkette Wahlwerbung $\rightarrow$ Emotionen $\rightarrow$ Einstellung analysiert, ist die Untersuchung von Chang (2001). Die Autorin weist im ersten Schritt nach, dass negative Werbung für einen politischen Kandidaten (die z. B. Kriminalität oder die nationale Sicherheit negativ darstellt) zu negativen affektiven Reaktionen führt. Umgekehrt löst positive Werbung (z. B. über Erfolge in der Wirtschaftspolitik oder Kriminalitätsbekämpfung) eher positive affektive Reaktionen aus. In einer nachfolgenden Mediationsanalyse weist die Autorin nach, dass die durch die Werbung ausgelösten positiven und negativen affektiven Reaktionen einen signifikanten Einfluss auf

2 Politische wie auch kommerzielle Werbung, die emotionale Appelle enthält, zielt sicher nicht immer nur auf eine Änderung der Einstellung ab, sondern kann auch dem Zweck dienen, Aufmerksamkeit für ein bestimmtes Thema oder einen Kandidaten zu erzeugen. Eine solche Wirkung wird jedoch im vorliegenden Zusammenhang nicht weiter berücksichtigt (vgl. hierzu etwa Miller 2007). 
die Einstellung zu dem jeweiligen politischen Kandidaten ausüben. Dieses Ergebnis ist allerdings in mindestens dreierlei Hinsicht eingeschränkt: Erstens handelt es sich im vorliegenden Experiment um Werbung für einen unbekannten politischen Kandidaten. Dies schränkt nach Meinung der Autorin den Geltungsbereich der Studie auf Situationen ein, in denen Kandidaten noch wenig bekannt seien, z. B. zu Beginn politischer Kampagnen. Dies bestätigen auch andere Studien: So werden affektive Reaktionen eher ausgelöst, wenn ein Einstellungsobjekt unbekannt ist (Thorson et al 1991), wenn nur schwache Voreinstellungen zu einem Einstellungsobjekt (Lanzetta et al. 1985) oder geringes Vorwissen vorliegen (Hsu/Price 1993). Bei bekannten Einstellungsobjekten oder verfügbaren Voreinstellungen sind affektive Reaktionen offensichtlich weniger leicht nachweisbar und/oder sie haben keine weitergehenden Konsequenzen.

Zweitens handelt es sich um die Wirkung eines einzigen Spots. Über kumulative Wirkungen der mehrfachen Exposition kann demnach nichts gesagt werden. Darüber hinaus kann auch über die Langfristigkeit eines solchen Effekts nur spekuliert werden. Unklar bleibt ebenfalls, wie sich ein solcher Spot auswirken würde, wenn ihm ein konterkarierender Spot gefolgt wäre. Einige experimentelle Studien zeigen deutlich, dass affektive Reaktionen am ehesten durch die einseitige Darstellung eines Sachverhaltes ausgelöst werden, jedoch kaum in einer zweiseitigen Kommunikationssituation, bei der die Pro- und Contra-Argumentation halbwegs ausgeglichen ist (Cobb 2005; Gross 2008).

Drittens mag die Mediationsanalyse zwar rein statistisch belegen können, dass Werbung affektive Reaktionen auslöst, die dann die Einstellung beeinflussen. Dieses Mediationsmodell ist aber äquivalent mit dem folgenden Modell: Werbung $\rightarrow$ Einstellung $\rightarrow$ Affekt (vgl. MacCallum et al. 1993). Mit anderen Worten, die kausale Abfolge lässt sich mittels dieser Analyse nicht belegen. Die Kausalrichtung der Einflüsse lässt sich letztlich nur durch die Analyse weiterer zeitlich verzögerter Messungen bestimmen (Cole/Maxwell 2003). Insgesamt kann man auf Basis der experimentellen Untersuchungen resümieren, dass politische Werbung zumindest kurzfristig affektive Reaktionen im Publikum verursacht und vermutlich auch die Einstellung von Wählerinnen und Wählern zu politischen Kandidaten und Sachverhalten beeinflusst. Ein empirischer Test dieser Annahme im Verlauf einer realen Kampagne steht allerdings noch aus und soll mit der folgenden Studie unternommen werden.

\section{Theoretische Annahmen}

Die vorliegende Untersuchung geht davon aus, dass insbesondere die Werbung in politischen Kampagnen auf die Emotionen der Wählerinnen und Wähler abzielt. Ein wichtiger Grund dafür, warum Werbung eher Emotionen auslöst als redaktionelle Angebote, liegt vor allem in der größeren Gestaltungsmacht begründet. So können Kommunikationsstrategen Werbung nahezu beliebig mit Stilmitteln versehen, die Emotionen beim Publikum auslösen. Diese Freiheit in der Gestaltung ihrer Botschaften haben politische Akteure nicht bei redaktionellen Angeboten. Selbst wenn politische Akteure Journalisten mit PR-Material versorgen, dürfte dieses Material redigiert, modifiziert oder zum Teil auch konterkariert werden. Dementsprechend zeigt sich auch in Inhaltsanalysen, dass ein Großteil des redaktionellen Medienangebots nicht emotionalisiert ist und damit auch weniger als Werbung geeignet sein dürfte, Emotionen beim Publikum auszulösen. Inhaltsanalysen für das deutsche Fernsehen zeigen, dass etwa vier Fünftel des Programms nicht emotionalisiert sind (Donsbach/Büttner 2005; Krüger/Zapf-Schramm 2003). Im Unterschied dazu enthält Wahlwerbung wesentlich häufiger Merkmale von Emotionalisierung (Kaid/Holtz-Bacha 2006). 
Aus diesem Grund untersucht die vorliegende Studie den Affekteinfluss der politischen Kampagnenwerbung und nicht von redaktionellen Angeboten. In Anlehnung an die bisherige Forschung wird davon ausgegangen, dass politische Werbung affektive Reaktionen in der Kampagne verursacht. Dabei wird angenommen, dass Werbung sowohl positive als auch negative Affekte beim Publikum auslöst (Hypothesen 1a und 1b). Diese negativen und positiven affektiven Reaktionen beeinflussen dann in einem weiteren Schritt die Einstellung der Rezipienten (Hypothesen 2a und 2b).

Die bisherige experimentelle Forschung hat dabei auf unterschiedliche Mechanismen hingewiesen, durch welche Prozesse der Affekteinfluss auf die Einstellung erfolgen kann. So zeigt eine Reihe von Studien in der Tradition der Zwei-Prozess-Modelle, dass affektive Reaktionen Einstellungen sowohl bei peripherer als auch bei zentraler Informationsverarbeitung beeinflussen können (Forgas 1995; Petty et al. 1993). Im ersten Fall dienen affektive Reaktionen als Heuristik für die Urteilsbildung, im zweiten Fall beeinflusst die Valenz der jeweiligen Affekte (d. h. positiv oder negativ) die Valenz der kognitiven Reaktionen und prägt auf diese Weise die Einstellung von Individuen. Im vorliegenden Fall ist lediglich der Effekt von affektiven Reaktionen auf die Einstellung relevant und weniger, welcher Prozess diesem Effekt unterliegt.

\section{Methode}

\subsection{Stichprobe und Durchfübrung der Befragung}

Um diese theoretischen Annahmen zu überprüfen, wurde eine dreiwellige, telefonische Panelbefragung in der Schweiz zur Abstimmung über die Verschärfung des Asylgesetzes durchgeführt. Die Stichprobe $(\mathrm{N}=1094$ in der dritten Befragungswelle) ist repräsentativ für die Bevölkerung der Deutsch- und Westschweiz (52 \% weiblich, $\mathrm{M}_{\text {Alter }}=48.18, \mathrm{SD}$ = 17.11; Bildung: $68 \%$ ohne Hochschul- bzw. Fachhochschulreife). Die erste Befragungswelle erfolgte von Ende Juni bis Anfang Juli 2006, die zweite Abfrage zum Höhepunkt der Kampagne Ende August 2006 und die dritte nach dem Abstimmungssonntag am 24.09.2006.

Dieses Referendum kam auf Initiative der politischen Linken zustande, weil ihrer Ansicht nach die humanitäre Tradition der Schweiz durch eine Asylgesetzverschärfung aufs Spiel gesetzt würde und dazu führte, dass wirklich Verfolgte kein Asyl mehr in der Schweiz bekämen. Demgegenüber argumentierten insbesondere rechtskonservative Akteure, dass es im Asylwesen zu viel Missbrauch gebe und dass viele Asylsuchende Kriminelle und Asylbetrüger seien. Beide Lager zielten auch durch Einzelfallgeschichten und Instrumentalisierung von Asylsuchenden auf die Emotionen der Wählerinnen und Wähler. Die Befürworter der Verschärfung beabsichtigten, mit der Darstellung von Asylsuchenden als Kriminelle und Betrüger negative Emotionen auszulösen, während die Gegner durch die Darstellung von Asylsuchenden als Opfer von Krieg und Folter positive Emotionen $\mathrm{zu}$ erregen suchten (vgl. zu dieser Kampagne genauer Schemer 2009a: 128f.).

Eine Inhaltsanalyse von Inseraten in den reichweitestärksten Zeitungen ${ }^{3}$ verdeutlicht, dass die Proponenten insgesamt stärker in politische Werbung investierten. Das Vorge-

3 Dazu gehörten folgende Informationsmedien: Blick, Sonntagsblick, Neue Zürcher Zeitung, NZZ am Sonntag, Tagesanzeiger, Sonntagszeitung, 20 Minuten, Aargauer Zeitung, Basler Zeitung, Berner Zeitung, Neue Luzerner Zeitung, Die Südostschweiz, St. Galler Tagblatt, Le Matin, Le Temps, Tribune de Genève, 24 heures, 20 Minutes, L’Hebdo, Punkt CH, Sonntag (AZ), Die Südostschweiz am Sonntag, Le Matin Dimanche. 
hen der Gegner erklärt sich wie folgt: Aufgrund der Knappheit an finanziellen Mitteln setzten die Gegner verstärkt auf Ereignisse und Pressemitteilungen, die dann in den Zeitungen und TV-Nachrichten aufgegriffen wurden (vgl. hierzu auch Schemer 2009a). Anhang 1 zeigt jeweils zwei Bilder, die die Plakat- bzw. Inseratmotive repräsentieren. Zwischen der ersten und zweiten Befragungswelle stammten 82 Prozent aller Zeitungsinserate von Verschärfungsbefürwortern. Im darauf folgenden Untersuchungszeitraum (zwischen Panelwelle 2 und 3) sprachen sich immer noch 59 Prozent der Inserate für die Asylgesetzverschärfung aus. Mit 44 Prozent der Pro-Inserate dominierte das Thema Asylmissbrauch und Kriminalität von Asylsuchenden die Werbekampagne der Verschärfungsbefürworter im ersten Zeitraum (48\% im Folgezeitraum). Die Gegner der Verschärfung sprachen zwischen der ersten und zweiten Panelwelle der Asylgesetzverschärfung die beabsichtigte Wirkung ab. Bei insgesamt nur 78 Inseraten kann jedoch nicht von einer koordinierten Werbekampagne gesprochen werden. Später stellten die Gegner einer Asylgesetzverschärfung das Thema humanitäre Tradition und das Menschenrecht auf Asyl in den Vordergrund (33\% aller Contra-Inserate). Damit standen in den Kampagnen der Proponenten wie auch der Kontrahenten Themen im Zentrum der strategischen Kommunikation, die mit großer Wahrscheinlichkeit Emotionen bei Wählerinnen und Wählern auszulösen vermögen (vgl. hierzu Abschnitt 3.2).

\subsection{Messung}

Um den Einfluss der politischen Werbung auf Affekte zu untersuchen, wurden positive und negative Emotionen gegenüber Asylsuchenden abgefragt. Als negative Emotionen gegenüber Asylsuchenden wurden drei Items gewählt: Angst, Ärger und Unbehagen (Cronbachs $\alpha=.73$ in der ersten, .76 in der zweiten und .78 in der dritten Welle). Als positive Emotionen wurden ebenfalls drei Items abgefragt: Freude, Hoffnung und Stolz (Cronbachs $\alpha=.72$ in der ersten, .78 in der zweiten und .79 in der dritten Welle). ${ }^{4}$ Die Einstellung zum Asylgesetz wurde mit zwei Items erfasst (z. B. „Ich bin für eine Verschärfung des Asylgesetzes“, Skala 1 „stimme voll und ganz zu“ bis 5 „stimme überhaupt

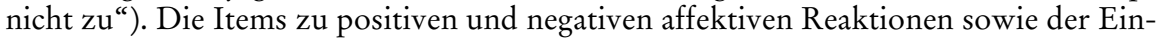
stellung wurden als latente Variablen modelliert (siehe 5.3).

Um den Einfluss der Werbung in der politischen Kampagne nachzuweisen, wurden die Befragten nach der Wichtigkeit unterschiedlicher Informationsquellen gefragt. Dabei wurde im Interview gefragt, wie wichtig politische Werbung jeweils in Inseraten und Plakaten für die Befragten ist, um Informationen über die Kampagne zu erhalten (Skala von 1 „sehr unwichtig“ bis 5 „sehr wichtig“). Diese beiden Items (Wichtigkeit von Inseraten und Plakaten) wurden in der ersten und zweiten Welle abgefragt (Korrelation

4 Der genaue Wortlaut für die Abfrage von Emotionen war: „Es geht ja nicht immer nur darum, was man denkt, sondern auch, wie man sich dabei fühlt. Wir möchten nun von Ihnen wissen, welche Gefühle Sie empfinden, wenn Sie an Asylbewerber in der Schweiz denken. Ich lese Ihnen jetzt einige Gefühle vor. Geben Sie bitte mit Zahlen von 1, das heißt ,sehr schwach', bis 5, sehr stark', an, wie stark Sie diese Gefühle empfinden. Wenn ich an Asylbewerber in der Schweiz denke, empfinde ich ... Angst, Ärger, Hoffnung, Freude, Unbehagen, Stolz.“ 
zwischen Wichtigkeit von Inseraten und Plakaten $r=.57$ in W1, $r=.64$ in W2)..$^{5}$ Anhang 2 zeigt die Mittelwerte und Standardabweichungen der Indikatoren bzw. deren Korrelationen untereinander.

Als Kontrollvariablen wurden analysiert: Geschlecht, Alter, Bildung, Links-RechtsSelbsteinschätzung (zehnstufige Skala von 1 „links“ bis 10 „rechts“). Die Kontrollvariablen wurden zum ersten Messzeitpunkt erhoben. Die Einflüsse der Kontrollvariablen wurden für alle Analysen berücksichtigt, werden aber im Folgenden nicht weiter ausgewiesen.

\subsection{Datenanalyse}

Die Daten werden auf der Basis von Strukturgleichungsmodellen mit latenten Variablen analysiert. Positive und negative Emotionen werden dabei als latente Variable modelliert, die die Ausprägung ihrer Indikatoren (jeweils drei) beeinflussen. Positive und negative Affekte werden in einem Modell mit zwei parallelen linearen Wachstumsprozessen modelliert (vgl. Schemer 2009b). Ein dritter Wachstumsprozess wird über das Einstellungskonstrukt modelliert. Abbildung 1 verdeutlicht das Modell inklusive der angenommenen Medienwirkungen. Dieses Parallelprozessmodell erlaubt neben der Analyse von Affekteinflüssen über die Zeit auch einen Test, ob sich positive und negative Affekte und die Einstellung zum Asylgesetz verändern und ob diese Veränderungen parallel verlaufen (vgl. allgemein zu Wachstumskurvenmodellen Bollen/Curran 2006; Duncan et al. 2006).

Dabei werden positive und negative Affekte und die Einstellung zum Asylgesetz als latente Variable zu drei Zeitpunkten modelliert und jeweils auf eine Konstante (Intercept-Faktor) und einen Steigungsfaktor (Slope) zurückgeführt. Die Pfade vom Intercept zu den Emotionen bzw. zur Einstellung werden jeweils alle auf 1 fixiert. Dadurch wird das Ausgangsniveau der Wachstumsdynamik repräsentiert. Die Dynamik des Wachstums wird durch die Fixierung der Pfade vom Steigungsfaktor zu den Affekt- bzw. Einstellungsfaktoren der jeweiligen Befragungswelle festgelegt. Bei einem linearen Anstieg werden diese Pfade auf 0,1 und 2 festgesetzt.

Die Medienwirkungen, die im vorliegenden Fall von Interesse sind, sind in diesem Modell mit Hilfe der Koeffizienten dargestellt. Von zentralem Interesse sind dabei insbesondere die Effekte a*g bzw. $\mathrm{c}^{*} \mathrm{~h}$, die die affektive Mediation des Einflusses politischer Werbung auf die Einstellung repräsentieren. Voraussetzung für diese Einflüsse sind aber zunächst statistisch bedeutsame Einflüsse der politischen Werbung auf positive und negative Affekte sowie signifikante Einflüsse von positiven und negativen Affekten auf die Einstellung der Befragten. Abbildung 1 verdeutlicht, dass die kausalen Einflüsse auf die

5 Statt der Häufigkeit der Exposition gegenüber politischer Werbung wurde die Wichtigkeit abgefragt, weil sich diese als bedeutsamer Indikator für Medienwirkungsstudien herausgestellt hat (z. B. Chaffee/Schleuder 1986). Die Wichtigkeit, die einem Angebot zugeschrieben wird, hängt in stärkerem Maße mit der Reizaufnahme zusammen und ist insofern ein Indikator für die Aufmerksamkeit, die einem bestimmten Reiz entgegengebracht wird (vgl. etwa McGuire 2001; Slater 2004). Insofern ist die selbstberichtete Häufigkeit der Mediennutzung ein distaler und die selbstberichtete Aufmerksamkeit ein proximaler Prädiktor für die vermeintliche Konfrontation mit einem bestimmten Medieninhalt. Beide Variablen korrelieren miteinander, weil in der Regel dasjenige Angebot als wichtig erachtet werden wird, welches häufiger genutzt wird. Umgekehrt folgt aber aus der häufigen Nutzung nicht zwangsläufig eine erhöhte Wichtigkeit oder Aufmerksamkeit, weil häufige Nutzung auch eine habituelle Nutzung bedeuten kann, die nicht zwangsläufig aufmerksamer sein muss. 
Einstellung nicht zeitgleich, sondern über die Zeit hinweg getestet werden, indem die jeweilige endogene Variable ( $\mathrm{z}$. B. negativer Affekt $_{\mathrm{t} 2}$ ) auf das jeweils zeitlich vorangehende verursachende Konstrukt (z. B. Nutzung politischer Werbung ${ }_{t 1}$ ) zurückgeführt wird. Dies ist dem Umstand geschuldet, dass Ursachen eine gewisse Zeit benötigen, um wirksam werden zu können (Cole/Maxwell 2003; Gollob/Reichardt 1987). Insbesondere Cole und Maxwell (2003) zeigen deutlich, dass Mediationseinflüsse von zeitgleich erhobenen Konstrukten nahezu immer verzerrte Schätzer wahrer Mediationseinflüsse über die Zeit darstellen. Die vorliegende Modellierung betrachtet daher nur zeitverzögerte Einflüsse, um die nachgewiesenen Einflüsse als kausale Effekte interpretieren zu können.

\section{Abbildung 1: Theoretisches Parallelprozessmodell zu Medienwirkungen auf positive und negative Affekte}

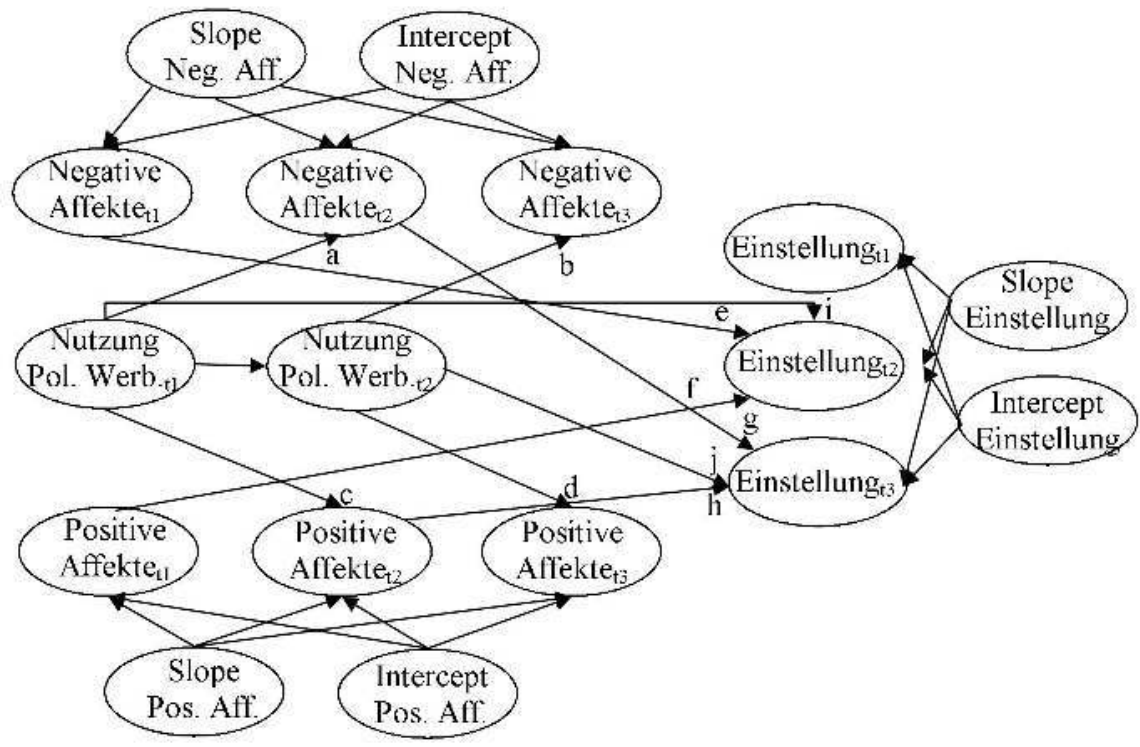

Anmerkungen: Ellipsen repräsentieren latente Variable, die die Ausprägung der Indikatoren (nicht dargestellt) verursachen; Kovarianzen zwischen latenten Variablen sind der Übersichtlichkeit halber nicht dargestellt.

a, b, c, d: Zeitverzögerte Einflüsse der politischen Werbung auf positive und negative Affekte. e, f, g, h: Zeitverzögerte Einflüsse der positiven und negativen Affekte auf die Einstellung. i, j: Zeitverzögerte direkte Einflüsse der politischen Werbung auf die Einstellung. $\mathrm{a}^{* \mathrm{~g}}, \mathrm{c}^{*} \mathrm{~h}$ : Zeitverzögerte Mediation des Einfluss der politischen Werbung auf die Einstellung durch negative und positive Affekte.

\section{Ergebnisse}

Insgesamt weist das Modell eine gute Passung an die Daten auf $\left(\chi^{2}=318.08, d f=295, p\right.$ $=.17, C F I=.99, R M S E A=.0190 \%-C I: .00-.01)$. Zunächst sollen deskriptiv die Befunde zur Veränderung positiver und negativer Emotionen im Kampagnenverlauf dargestellt werden, bevor auf die Einflüsse der politischen Werbung eingegangen wird. Die Mit- 
telwerte der Steigungsfaktoren der negativen Affekte $(M=.099, p<.05)$ und der Einstellung zum Asylgesetz $(M=-.144, p<.05)$ sind signifikant von Null verschieden, nicht aber der Mittelwert des Steigungsfaktors der positiven Affekte $(M=.011, n s)$. Das bedeutet einen linearen Anstieg der negativen affektiven Reaktionen, wie Angst oder Ärger gegenüber Asylsuchenden, sowie eine lineare Änderung der Einstellung in Richtung Befürwortung der Asylgesetzverschärfung. Die positiven affektiven Reaktionen der Befragten ändern sich jedoch offensichtlich nicht linear. Sowohl bei positiven $\left(\sigma^{2}=.026\right.$, $p<.05)$ und negativen affektiven Reaktionen $\left(\sigma^{2}=.017, p<.05\right)$ sowie bei der Einstellung $\left(\sigma^{2}=.054, p<.01\right)$ liegen signifikante interindividuelle Unterschiede in der zeitlichen Entwicklung vor. D. h. es gibt Personen, die von diesem linearen Trend abweichen. Dasselbe trifft auf das Anfangsniveau der positiven und negativen Affekte sowie der Einstellung zu. Die signifikant von Null verschiedenen Varianzen der Konstanten (Einstellung: $\sigma^{2}=1.127, p<.01$; negative Affekte: $\sigma^{2}=.592, p<.01$; positive Affekte: $\sigma^{2}=$. $366, p<.01$ ) deuten auf interindividuelle Unterschiede bei den Befragten hin, die unabhängig von der Kampagne bereits bestehen. ${ }^{6}$

Anhand der Korrelationen zwischen den Wachstumsfaktoren lassen sich folgende Ergebnisse ablesen: Die Konstanten des positiven und negativen Affekts und der Einstellung korrelieren alle signifikant miteinander $\left(r_{\text {Affekt pos.-neg. }}=-.44, r_{\text {Affekt pos.-Einstellung }}\right.$ $=.50, r_{\text {Affekt neg.-Einstellung }}=-.73$ ). Diese Korrelationen bedeuten, dass eine anfänglich hohe Ausprägung von positiven Affekten gegenüber Asylsuchenden mit einer geringen Ausprägung von negativen Affekten zu Beginn einhergeht und mit einer anfänglichen Ablehnung der Asylgesetzverschärfung. Eine anfänglich hohe Ausprägung von negativen Emotionen steht mit einer Ablehnung der Verschärfung zu Beginn der Kampagne in Zusammenhang. Der Einstellungs-Intercept korreliert darüber hinaus mit dem Einstellungs-Slope $(r=.22)$. D. h. je eher Rezipienten zu Beginn die Verschärfung befürworten, desto stärker polarisiert sich ihre Einstellung im weiteren Verlauf. Dasselbe trifft auch auf die anfänglichen Gegner zu. Die Slope-Faktoren von positiven und negativen Affekten sowie der Einstellung kovariieren nicht signifikant miteinander. Die Veränderung von positiven und negativen Affekten und der Einstellung erfolgt demnach nicht parallel. Insgesamt zeigt sich also eine Dynamik und Varianz vor allem bei den negativen Affekten und der Einstellung der Befragten, die durch die Mediennutzung erklärt werden kann.

In der ersten Hypothese wurde angenommen, dass politische Werbung positive und negative affektive Reaktionen beim Publikum auslöst. Abbildung 2 stellt die Ergebnisse der Medienwirkungsanalyse dar. Dabei handelt es sich um Pfadkoeffizienten aus dem in Abb. 1 dargestellten Modell. Abbildung 2 verdeutlicht, dass politische Werbung in beiden Erhebungszeiträumen zwar verstärkt negative Affekte erzeugt, aber nicht zu mehr positiven affektiven Reaktionen beim Publikum führt. Mit anderen Worten, die Aufmerksamkeit für politische Werbung in der ersten und zweiten Befragungswelle führt bei Rezipienten zu mehr Angst und Ärger gegenüber Asylsuchenden zum jeweils darauf folgenden Befragungszeitpunkt. Gleichzeitig hat die Aufmerksamkeit gegenüber politischer Werbung keinen Einfluss auf Hoffnung, Stolz oder Freude im Zusammenhang mit Asylsuchenden. Dies bestätigt die erste Annahme zumindest partiell.

Betrachtet man die Wirkungen der politischen Werbung auf negative Affekte genauer, dann kann man einen signifikanten Anstieg in der Wirkung über die Zeit feststellen $(z=2.11, p<.05)$. Die Werbewirkung zum Zeitpunkt t 2 auf die Affekte zum Zeitpunkt t3 ist also stärker als im ersten Zeitraum. Dies ist insofern plausibel, als das Aufkommen politischer Werbung zwischen dem zweiten und dritten Befragungszeitpunkt höher war

6 Die Mittelwerte der Konstanten wurden zur Identifikation des Modells auf 0 fixiert. 
Abbildung 2: Der Einfluss der politischen Werbung auf positive und negative Affekte und die Einstellung im Zeitverlauf

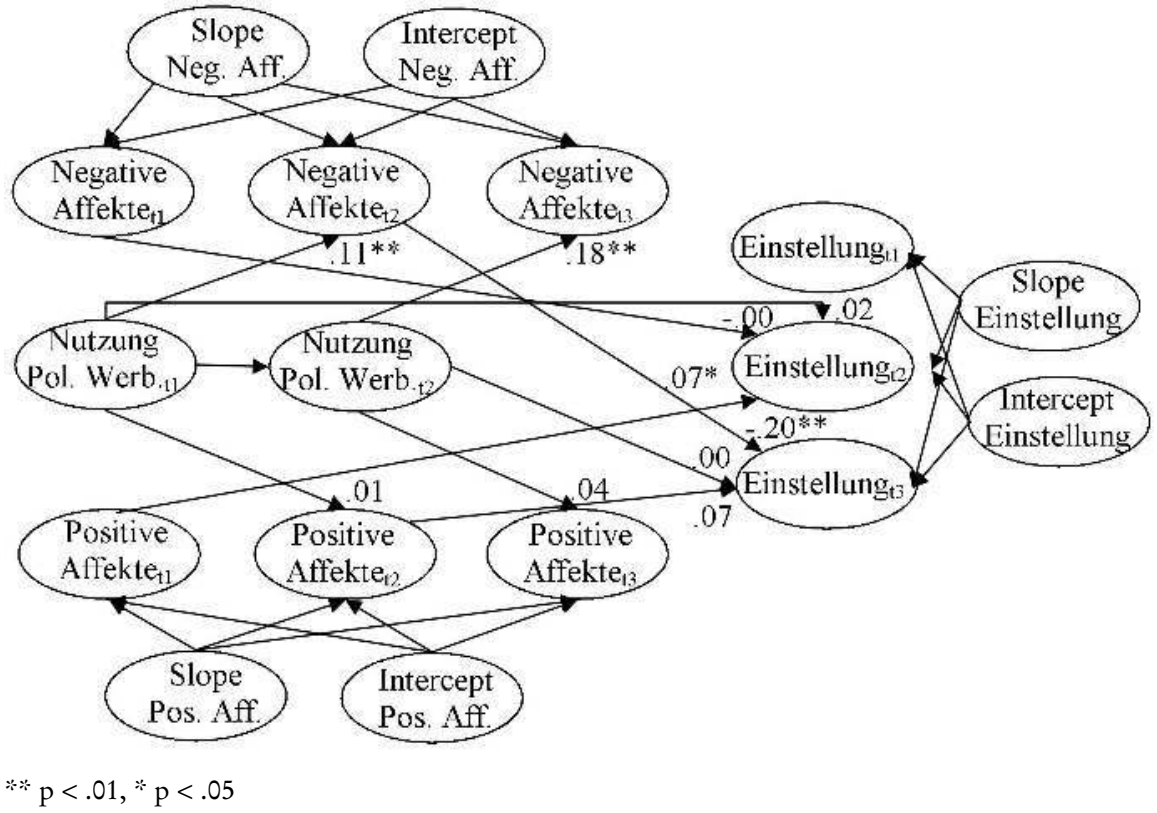

als noch zum Beginn der Kampagne. Schließlich verdeutlicht Abbildung 2, dass es keinen direkten Effekt der Werbung auf die Einstellung gibt.

In der zweiten Hypothese wurde angenommen, dass positive und negative Affekte den Einfluss der Werbung auf die Einstellung mediieren. Voraussetzung für diesen Mediationseinfluss ist zunächst aber, dass die affektiven Reaktionen die Einstellung beeinflussen. Die Wirkungen von positiven und negativen Affekten auf die Einstellung können ebenfalls Abbildung 2 entnommen werden. Positive Affekte verringern zum ersten Zeitpunkt der Befragung die Zustimmung zur Asylgesetzverschärfung, im folgenden Zeitraum aber nicht mehr. Im Unterschied dazu leisten negative Affekte in der ersten Befragungswelle keinen signifikanten Erklärungsbeitrag zur Einstellung in der darauffolgenden Welle. Negative Affekte werden aber gegen Ende der Kampagne zu einer bedeutsamen Erklärungsgröße für die Einstellung. Je negativer die affektiven Reaktionen der Befragten sind, desto eher sind sie für die Verschärfung der Asylgesetzgebung.

Der angenommene Mediationseffekt ergibt sich nun aus dem Einfluss der Werbung auf positive und negative Affekte sowie aus der Affektwirkung auf die Einstellung. Da nur Effekte über die Zeit geschätzt werden, gibt es nur zwei Mediationseinflüsse, die in Abb. 1 mit a*g (Werbung $_{\mathrm{t} 1} \rightarrow$ positive Affekte $_{\mathrm{t} 2} \rightarrow$ Einstellung $_{\mathrm{t} 3}$ ) und $\mathrm{c}^{* \mathrm{~h}}\left(\right.$ Werbung $_{\mathrm{t} 1}$ $\rightarrow$ negative Affekte $_{\mathrm{t} 2} \rightarrow$ Einstellung $_{\mathrm{t} 3}$ ) bezeichnet sind. Bei der Signifikanzprüfung dieser Mediationseffekte zeigt sich, dass der Mediationseffekt „Werbung ${ }_{\mathrm{t} 1} \rightarrow$ positive Affekte $_{\mathrm{t} 2}$ $\rightarrow$ Einstellung $_{\mathrm{t} 3}$ " nicht signifikant von Null verschieden ist $(z<1)$. Dies liegt vor allem daran, dass positive Affekte überhaupt nicht durch die Werbung erzeugt werden bzw. später keinen prädiktiven Wert für die Einstellung haben. Der Mediationseffekt „Werbung $_{\mathrm{t} 1} \rightarrow$ negative Affekte $_{\mathrm{t} 2} \rightarrow$ Einstellung $_{\mathrm{t} 3}$ " ist jedoch signifikant $(z=-2.09, p<.05)$. Das bedeutet, dass die durch die Werbung verursachten negativen Affekte auch die Ein- 
stellung beeinflussen. Wenn also politische Werbung Angst und Ärger gegenüber Asylsuchenden in der Kampagne auslöst, dann hat dies bei Rezipienten eine verstärkte $\mathrm{Zu}$ stimmung zur Asylgesetzverschärfung zur Konsequenz. Dieses Ergebnis bestätigt die Mediationsannahme zumindest für negative Affekte als Mediatoren der Wirkung politischer Werbung auf die Einstellung von Rezipienten.

\section{Diskussion}

Die vorliegende Studie zeigt deutlich, dass die durch die Werbekampagne verursachten Emotionen die Einstellung von Rezipienten zu einem politischen Einstellungsobjekt hier die Vorlage zur Verschärfung des Asylgesetzes - beeinflussen können. Dieser Mediationseffekt ließ sich jedoch nur für negative Affekte, z. B. Angst oder Ärger, nachweisen, nicht jedoch für positive Affekte, wie Freude oder Hoffnung. D. h. im Verlauf der Kampagne wurden die negativen Affekte der Wählerinnen und Wähler zunehmend negativer, und dies lässt sich auf die Wirkungen der Werbekampagne zurückführen. Gleichzeitig nahm die Zustimmung zur Verschärfung im Zeitverlauf zu, und die durch die politische Werbekampagne verursachten negativen Affekte lieferten einen signifikanten Erklärungsbeitrag zu dieser Änderung. Bezogen auf die konkrete Kampagne bedeutet dies, dass es den Befürwortern der Asylgesetzverschärfung also durch ihre Werbekampagne eher als den Gegnern gelang, ihr Ziel - nämlich die Unterstützung für die Asylgesetzverschärfung - in der Bevölkerung zu erreichen.

Dabei stellt die vorliegende Modellierung sicher, dass es sich um kausal interpretierbare Kampagnenwirkungen handelt. ${ }^{7}$ Es wurden lediglich diejenigen Mediationswirkungen untersucht, bei denen die Ursache der Wirkung aufgrund der gewählten Befragungszeitpunkte vorausgeht. Damit bestätigt das vorliegende Ergebnis zumindest in Bezug auf negative affektive Mediation des Werbeeinflusses auf die Einstellung die Befunde, die sich auch bereits in Laboruntersuchungen gezeigt haben (Chang 2001). Die vorliegenden Ergebnisse gehen in zweierlei Hinsicht über die bisherigen Erkenntnisse zur affektiven Wirkung von politischer Werbung hinaus. Erstens zeigen sie, dass affektive Wirkungen im Verlauf realer Kampagnen unter zweiseitigen Kommunikationsbedingungen auftreten und nicht lediglich Kurzzeitphänomene sind, die sich im Experimentallabor herstellen lassen. Zweitens lässt sich durch die vorliegende Modellierung die Kausalabfolge politische Werbung $\rightarrow$ negative Emotionen $\rightarrow$ Einstellung sehr gut veranschaulichen. Dies im Unterschied zu den bisherigen Studien, bei denen mindestens zwei Konstrukte zeitgleich erhoben wurden und daher die Kausalabfolge nicht zweifelsfrei bestimmt werden konnte (vgl. auch Cole/Maxwell 2003). Insofern kann die vorliegende Studie auch als eine Art der Validierung für die Befunde aus Laborexperimenten bzw. auch aus vorigen Befragungsstudien gesehen werden.

Drei Aspekte sind jedoch diskussionswürdig: Der erste betrifft den Befund, dass keine positiven Mediationswirkungen nachgewiesen werden konnten. Der zweite Aspekt betrifft die Stärke der Mediationseffekte von politischer Werbung über negative Affekte auf die Einstellung. Der dritte Aspekt bezieht sich auf die Verallgemeinerbarkeit der vorliegenden Ergebnisse. Zum ersten Aspekt: Zwar zeigt sich in den Analysen und in Übereinstimmung mit bisherigen Befragungsstudien in US-Wahlkämpfen, dass po-

7 Kausalität impliziert neben der Korrelation von Konstrukten und deren bestimmbarer zeitlicher Abfolge auch die Abwesenheit von dritten Variablen, die für den Zusammenhang der Konstrukte verantwortlich sein könnten. Die ersten beiden Bedingungen werden durch die vorliegende Untersuchung erfüllt. Die letzte Bedingung lässt sich jedoch nur in Experimenten zweifelsfrei erfüllen. 
sitive und negative Affekte die Einstellung von Befragten beeinflussen. In dieser Panelstudie wurden jedoch die positiven affektiven Reaktionen nicht durch die politische Werbung verursacht. Eine Erklärung dafür wäre, dass bei der politischen Werbung in der Asylgesetzkampagne das Pro-Verschärfungs-Lager klar dominierte. Dieses verfügte eher über finanzielle Mittel zur Schaltung von Inseraten und Plakaten. Eine Inhaltsanalyse der politischen Werbung in Zeitungen verdeutlicht das Übergewicht der Proponenten in der Asylgesetzkampagne: 80 \% der Werbung in Inseraten ging auf die Proponenten der Asylgesetzverschärfung zurück. Das Argument, das dabei am häufigsten genannt wurde, thematisierte Asylmissbrauch, den es durch die Verschärfung des Gesetzes einzudämmen galt. Angesichts der Übermacht von Werbung, die das Thema Asylmissbrauch propagierte, ist zumindest die Entstehung von negativen Affekten gegenüber Asylsuchenden wenig überraschend. Weil die Gegner der Verschärfung nicht über die finanziellen Mittel verfügten, um in Werbung zu investieren, konnten sie diesem negativen Bild von Asylbewerbern nicht mit positiver Werbung entgegenwirken und positive Emotionen bei den Rezipienten erzeugen. Das bedeutet jedoch nicht, dass die Kampagne überhaupt keine positiven Emotionen hervorgerufen hätte. Wenn auch nicht durch werbevermittelte Kommunikation, so doch zumindest über die Berichterstattung über Flüchtlingsschicksale und über die Verpflichtung, die humanitäre Tradition der Schweiz zu wahren, gelang es den Gegnern der Asylgesetzverschärfung, positive Emotionen bei den Rezipienten wachzurufen (Schemer 2009a). ${ }^{8}$

Der zweite Aspekt, der vertiefend diskutiert werden soll, betrifft die Stärke der nachgewiesenen Mediationswirkungen. Die hier nachgewiesenen Medienwirkungen auf Emotionen und Einstellungen fallen insgesamt eher gering aus. Dieses Ergebnis ist zum einen darauf zurückzuführen, dass die emotionalen Reaktionen der Befragten nicht auf die tatsächlich genutzten Medieninhalte zurückgeführt wurden, sondern auf die Mediennutzung. Während der Mediennutzung werden aber nicht nur affektive Reaktionen ausgelöst, sondern auch kognitive. Diese kognitiven Reaktionen können Emotionen aber auch abschwächen (Petty et al. 1993). Führt man affektive Reaktionen lediglich auf die Mediennutzung zurück und nicht auf die Affekt auslösenden Inhalte, dann dürfte die Vorhersage affektiver Reaktionen also schwächer ausfallen. Zum anderen sind die Effekte vermutlich auch deshalb gering, weil zwischen Ursache (d. h. der konkreten Mediennutzungssituation) und der Medienwirkung mehrere Tage oder sogar Wochen liegen. Sprich: Der tatsächliche Wirkungszeitraum entspricht nicht dem vorliegenden Erhebungszeitraum (vgl. auch Slater 2007).

Eine dritte Ursache für die eher geringen Effekte kann aber auch die mangelnde Berücksichtigung von Variablen sein, die Rezipienten für stärkere Werbewirkungen auf

8 Die Frage, welchen Anteil relativ gesehen die politische Werbung an der Emotionserregung im Vergleich zur Medienberichterstattung über das Asylgesetz hatte, kann im vorliegenden Beitrag nicht abschließend beantwortet werden. Allerdings deutet das Muster der Ergebnisse eindeutig auf Werbewirkungen. Es konnten nur Einflüsse der Aufmerksamkeit für politische Werbung auf negative Emotionen nachgewiesen werden, aber nicht auf positive. Dies ist darauf zurückzuführen, dass die Werbekampagne durch eine Dominanz der Befürworter einer Asylgesetzverschärfung gekennzeichnet war. Die Berichterstattung über das Thema war jedoch durch eine Dominanz der Gegner charakterisiert. Diese Berichterstattung über die gegnerische Kampagne schwächte negative Emotionen ab und löste positive Emotionen aus (vgl. hierzu Schemer 2009a). Wenn die Werbewirkung im vorliegenden Fall mit der Wirkung der Berichterstattung konfundiert wäre, dann müsste man Abschwächungseffekte auf negative Emotionen und Stimulierungseffekte auf positive Emotionen finden. Dies war jedoch nicht der Fall. Dies erhärtet die Plausibilität der vorliegenden Werbewirkungsannahmen. 
Emotionen prädisponiert. So zeigen insbesondere Experimente, dass Affekteinflüsse stärker sind, wenn ein Einstellungsobjekt unbekannt ist (Thorson et al. 1991) oder schwache Voreinstellungen (Lanzetta et al. 1985) bzw. geringes Vorwissen vorliegen (Hsu/Price 1993). So könnten vertiefende Analysen der vorliegenden Daten durchgeführt werden, die die Bedeutung dieser Randbedingungen für die Stärke affektive Werbewirkungen näher untersuchen.

Man könnte daher für die vorliegende Studie annehmen, dass möglicherweise situativ stärkere affektive Wirkungen der politischen Werbung aufgetreten sind. Dies würde bedeuten, dass die in der vorliegenden Untersuchung nachgewiesenen Einflüsse lediglich Residualwirkungen ursprünglich stärkerer Effekte sind. Ob durch das vorliegende Vorgehen wahre Wirkungen tatsächlich unterschätzt werden, kann jedoch nicht abschließend beurteilt werden, sondern es handelt sich lediglich um eine plausible Annahme, die weiterer Forschung bedarf. Hierzu müssten zu ein und derselben Kampagne sowohl eine Längsschnittbefragung mit begleitender Inhaltsanalyse und mindestens eine experimentelle Studie zur Validierung der Medienwirkungsergebnisse aus der Felduntersuchung durchgeführt werden.

Der letzte wichtige Diskussionspunkt betrifft die Verallgemeinerbarkeit der vorliegenden Ergebnisse, insbesondere vor dem Hintergrund, dass die vorliegende Studie im Rahmen einer Kampagne zum Asylgesetz in der Schweiz stattfand. Allerdings bestätigt die Untersuchung ein Muster von Ergebnissen, die auch in anderen Ländern im Zusammenhang mit ähnlichen Themen nachgewiesen wurden. Demnach scheinen die vorliegenden Ergebnisse typisch zu sein für die Wirkungen von Medienberichten über ethnische Minderheiten oder Kampagnenkommunikation, die gezielt ethnische Minderheiten angreift. Wenn ethnische Minderheiten stigmatisiert werden (z. B. durch die Darstellung als Wirtschaftsflüchtlinge oder Kriminelle), dann löst dies negative Emotionen aus. Ein solcher Effekt zeigte sich in einer experimentellen Studie in Spanien (Igartua et al. 2007) wie auch in einer Reihe von Experimenten in den USA (z. B. Peffley et al. 1996).

Neben der negativen Thematisierung von ethnischen Minderheiten treten vergleichbare negative Affektwirkungen auch auf bei der Darstellung von Kriegen (Schoen 2006; Pan/Kosicki 1994) bzw. Katastrophen, wie etwa die Anschläge auf das World Trade Center 2001 (Cho et al. 2003). Der vorliegende Kontext einer direktdemokratischen Abstimmung über eine Gesetzesvorlage in der Schweiz scheint daher kein Sonderfall zu sein, sondern typisch für die Wirkung negativer werthaltiger Themen. Mit anderen Worten: Sobald Kampagnen geführt werden, bei denen es politischen Akteuren gelingt, werthaltige Themen zu besetzen, die das Wahlvolk „bewegen“, dürften auch affektive Kampagnenwirkungen auf Einstellungen zu beobachten sein. Solche Wirkungen dürften umso stärker ausfallen, je einseitiger die Kommunikationssituation ausgeprägt ist. Damit ergänzt diese Untersuchung bisherige Studien, die vor allem Affekteinflüsse bei politischen Kandidaten untersucht haben.

Am Ende stellt sich auch die Frage, wie man diese medieninduzierten Affekteinflüsse auf die Einstellungen von Rezipienten bewertet und welche Implikationen die Emotionseinflüsse für den Umgang mit gesellschaftlichen Minderheiten, wie etwa Flüchtlingen, haben. Die Ergebnisse der vorliegenden Studie bestätigen nämlich die sehr pessimistische Sicht, dass insbesondere in Wohlfahrtsstaaten ein Bild von Flüchtlingen dominiert, das dazu führt, dass diese nicht mehr als menschliche Individuen wahrgenommen werden (Esses et al. 2008). Einerseits werden Flüchtlinge als Masse dargestellt, die westliche Wohlfahrtsstaaten wie Naturkatastrophen treffen. Wenn Flüchtlinge als Individuen dargestellt werden, dann als Betrüger und Kriminelle, deren böse Absicht die Gutmütigkeit und Hilfsbereitschaft der Gastgeberländer unterminiert. In beiden Fällen 
resultieren Ressentiments und eine Feindschaft gegenüber Flüchtlingen, die den politischen Status Quo zementiert und restriktivere politische Programme gegenüber Minderheiten legitimiert. Insbesondere rechtspopulistische Parteien haben diese Situation für ihre Zwecke ausgeschlachtet und bewirtschaften solche Themen zur Stimmenmaximierung nicht mehr nur zu Wahlkampfzeiten (vgl. Boomgarden/Vliegenthart 2007). Zu diesen rechtspopulistischen Parteien gesellen sich aber immer mehr bürgerliche Parteien, die ihre Felle davonschwimmen sehen und ebenfalls mit Ausländer- und Minderheitenthemen auf Stimmenfang gehen. Auf diese Weise kommt eine Spirale in Gang, die ein Verständnis für die Notlage wirklicher Flüchtlinge und Minderheiten erheblich erschwert.

\section{Literatur}

Abelson, Robert P./Kinder, Donald R./Peters, Mark D./Fiske, Susan T. (1982): Affective and Semantic Components in Political Person Perception. In: Journal of Personality and Social Psychology, 42. Jg., Heft 4, 619-630.

Bollen, Kenneth A./Curran, Patrick J. (2006): Latent Curve Models. Hoboken, NJ: Wiley.

Boomgarden, Hajo G./Vliegenthart, Rens (2007): Explaining the Rise of Anti-Immigrant Parties: The Role of News Media Content. In: Electoral Studies, 26. Jg., 404-417.

Brader, Ted (2005): Striking a Responsive Chord: How Political Ads Motivate and Persuade Voters by Appealing to Emotions. In: American Journal of Political Science, 49. Jg., Heft 2, 388-405.

Brader, Ted (2006): Campaigning for Hearts and Minds: How Emotional Appeals in Political Ads Work. Chicago: University of Chicago Press.

Breckler, Steven J. (1984): Empirical Validation of Affect, Behavior, and Cognition. In: Journal of Personality and Social Psychology, 47. Jg., Heft 6, 1191-1205.

Chaffee, Steven H./Schleuder, Joan (1986): Measurement and Effects of Attention to Media News. In: Human Communication Research, 13. Jg., 76-107.

Chang, Chingching (2001): The Impacts of Emotion Elicited by Print Political Advertising on Candidate Evaluation. In: Media Psychology, 3. Jg., 91-118.

Cho, Jaeho/Boyle, Michael P./Keum, Heejo/Shevy, Mark D./McLeod, Douglas M./Shah, Dhavan V./Pan, Zhongdang (2003): Media, Terrorism, and Emotionality: Emotional Differences in Media Content and Public Reactions to the September 11th Terrorist Attacks. In: Journal of Broadcasting and Electronic Media, 47. Jg., Heft 3, 309-327.

Cobb, Michael D. (2005): Framing Effects on Public Opinion about Nanotechnology. In: Science Communication, 27. Jg., Heft 2, 221-239.

Cole, David A./Maxwell, Scott E. (2003): Testing Mediational Models with Longitudinal Data: Questions and Tips in the Use of Structural Equation Modeling. In: Journal of Abnormal Psychology, 112. Jg., Heft 4, 558-577.

Dahlem, Stefan (2001). Wablentscheidung in der Mediengesellschaft. Theoretische und empirische Grundlagen der interdisziplinären Wablforschung. Freiburg: Alber.

Donsbach, Wolfgang/Büttner, Katrin (2005): Boulevardisierungstrend in deutschen Fernsehnachrichten. Darstellungsmerkmale der Politikberichterstattung vor den Bundestagswahlen 1983, 1990 und 1998. In: Publizistik, 50. Jg., Heft 1, 21-38.

Duncan, Terry E./Duncan, Susan C./Strycker, Lisa A. (2006): An Introduction to Latent Variable Growth Curve Modelling. Mahwah, NJ: Erlbaum.

Ellsworth, Phoebe C./Scherer, Klaus R. (2003): Appraisal Processes in Emotion. In: Davidson, Richard J./Scherer, Klaus R./Goldsmith, H. Hill (Hrsg.), Handbook of affective sciences. New York: Oxford University Press, 572-595.

Esses, Victoria M./Veenvliet, Scott/Hodson, Gordon/Mihic, Ljiljana (2008): Justice, Morality, and the Dehumanization of Refugees. In: Social Justice Research, 21. Jg., 4-25.

Forgas, Joseph P. (1995): Mood and Judgment: The Affect Infusion Model (AIM). In: Psychological Bulletin, 117. Jg., Heft 1, 39-66.

Gollob, Harry F./Reichardt, Charles S. (1987): Taking Account of Time Lags in Causal Models. In: Child Development, 58. Jg., 80-92. 
Gross, Kimberly (2008): Framing Persuasive Appeals: Episodic and Thematic Framing, Emotional Response, and Policy Opinion. In: Political Psychology, 29. Jg., Heft 2, 169-192.

Hartmann, George W. (1936): A Field Experiment on the Comparative Effectiveness of "Emotional" and "Rational" Political Leaflets in Determining Election Results. In: Journal of Abnormal and Social Psychology, 31. Jg., 99-114.

Hsu, Mei-Ling/Price, Vincent (1993): Political Expertise and Affect. In: Communication Research, 20. Jg., Heft 5, 671-695.

Igartua, Juan J./Otero, José/Muñiz, Carlos/Cheng, Lifen/Gómez, José (2007): Efectos cognitivos y afectivos de los encuadres noticiosos de la immigración. In: Igartua, Juan J./Muñiz, Carlos (Hrsg.), Medios de comunicación, inmigración y sociedad. Salamanca: Ediciones Universidad de Salamanca, 197-232.

Kaid, Lynda L. (2004): Political Advertising. In: Kaid, Lynda L. (Hrsg.), Handbook of Political Communication Research. Mahwah, NJ: Erlbaum, 155-202.

Kaid, Lynda L./Holtz-Bacha, Christina (1993): Audience Reactions to Televised Political Programs: An Experimental Study of the 1990 German National Election. In: European Journal of Communication, 8. Jg., 77-99.

Kaid, Lynda L./Holtz-Bacha, Christina (1995): Political Advertising Across Cultures. Comparing Content, Styles, and effects. In: Kaid, Lynda L./Holtz-Bacha, Christina (Hrsg.), Political Advertising in Western Democracies: Parties and Candidates on Relevision. Thousand Oaks, CA: Sage, 206-227.

Kaid, Lynda L./Holtz-Bacha, Christina (2006): Television Advertsing and Democratic Systems Around the World. In: Kaid, Lynda L./Holtz-Bacha, Christina (Hrsg.), The Sage Handbook of Political Advertising. Thousand Oaks, CA: Sage, 445-457.

Kaid, Lynda L./Johnston, Anne (2001): Videostyle in Presidential Campaigns. Style and Content of Televised Political Advertising. Westport: Praeger.

Krüger, Udo M./Zapf-Schramm, Thomas (2003): Inhalte und Gestaltung öffentlicher und privater Informationsangebote im Fernsehen. In: Media Perspektiven, 12. Jg., 534-548.

Lanzetta, John T./Sullivan, Denis G./Masters, Roger D./McHugo, Gregory J. (1985): Emotional and Cognitive Responses to Televised Images of Political Leaders. In: Kraus, Sidney/Perloff, Richard M. (Hrsg.), Mass Media and Political Thought. An Information-Processing Approach. Beverly-Hills: Sage, 85-116.

Lazarsfeld, Paul F./Berelson, Bernard/Gaudet, Hazel (1944): The People's Choice: How the Voter Makes Up his Mind in a Presidential Campaign. New York: Columbia University Press.

MacCallum, Robert C./Wegener, Duane T./Uchino, Bert N./Fabrigar, Leandre R. (1993): The Problem of Equivalent Models in Application of Covariance Structure Analysis. In: Psychological Bulletin, 114. Jg., Heft 1, 185-199.

Maier, Jürgen/Maier, Michaela (2007): Audience Reactions to Negative Campaign Spots in the 2005 German National Elections. In: Human Communication, 10. Jg., Heft 3, 329-344.

Marcus, George E./MacKuen, Michael B. (1993): Anxiety, Enthusiasm, and the Vote: The Emotional Underpinnings of Learning and Involvement During Presidential Campaigns. In: American Political Science Review, 87. Jg., Heft 3, 672-685.

McGuire, William J. (2001): Theoretical Foundations of Campaigns. In: Rice, Ronald E./Atkin, Charles K. (Hrsg.), Public Communication Campaigns. Newbury Park: Sage, 43-66.

Miller, Joanne Marie (2007): Examining the Mediators of Agenda Setting: A New Experimental Paradigm Reveals the Role of Emotions. In: Political Psychology, 28. Jg., 689-717.

Otto, Jürgen H./Euler, Harald A./Mandl, Heinz (2000): Begriffsbestimmungen. In: Otto, Jürgen H./Euler, Harald A./Mandl, Heinz (Hrsg.), Emotionspsychologie. Ein Handbuch. Weinheim: Psychologie Verlags Union, 11-18.

Pan, Zhongdang/Kosicki, Gerald M. (1994): Voter's Reasoning Processes and Media Influences During the Persian Gulf War. In: Political Behavior, 16. Jg., Heft 1, 117-156.

Peffley, Mark/Shields, Todd G./Williams, Bruce (1996): The Intersection of Race and Crime in Television News Stories: An Experimental Study. In: Political Communication, 13. Jg., 309-327.

Petty, Richard E./Schumann, David W./Richman, S. A./Strathman, A. (1993): Positive Mood and Persuasion: Different Roles for Affect under High and Low Elaboration Conditions. In: Journal of Personality and Social Psychology, 64. Jg., 5-20. 
Rosenberg, Milton J./Hovland, Carl I. (1960): Cognitive, Affective, and Behavioural components of attitudes. In: Hovland, Carl I./Rosenberg, Milton J. (Hrsg.): Attitude Organization and Change. New Haven: Yale University Press, 1-14.

Ruhrmann, Georg/Woelke, Jens/Maier, Michaela/Diehlmann, Nicole (Eds.) (2003): Der Wert von Nachrichten im deutschen Fernsehen. Opladen: Leske und Budrich.

Schemer (2009a): Kampagnen für Herz und Verstand. Kognitive und affektive Einflüsse der Massenmedien auf politische Einstellungen. Baden-Baden: Nomos/Edition Reinhard Fischer.

Schemer, Christian (2009b): Wie Boulevardmedien Emotionen schüren. Der Einfluss der Mediennutzung auf Emotionen in politischen Kampagnen. In: Schemer, Christian; Wirth, Werner; Wünsch, Carsten (Hrsg.): Politische Kommunikation: Wahrnehmung, Verarbeitung, Wirkung. Baden-Baden: Nomos/Edition Reinhard Fischer, 133-152.

Scherer, Klaus R./Schorr, Angela/Johnstone, Tom (Eds.) (2001): Appraisal Processes in Emotion. Theory, Methods, Research. Oxford, New York: Oxford University Press.

Scheufele, Bertram/Gasteiger, Carolin (2007): Berichterstattung, Emotionen und politische Legitimierung. Eine experimentelle Untersuchung zum Einfluss der Politikberichterstattung auf die Legitimierung politischer Entscheidungen am Beispiel von Bundeswehreinsätzen. In: Medien E Kommunikationswissenschaft, 55. Jg., Heft 4, 534-554.

Schimmack, Ulrich/Crites, Stephen L. (2005): The Structure of Affect. In: Albarracín, Dolores/ Johnson, Blair T./Zanna, Mark P. (Hrsg.), The Handbook of Attitudes. Mahwah: Erlbaum, 397-435.

Schmidt-Atzert, Lothar (2000): Struktur der Emotionen. In: Otto, Jürgen H./Euler, Harald A./ Mandl, Heinz (Hrsg.), Emotionspsychologie. Ein Handbuch. Weinheim: Psychologie Verlags Union, 30-44.

Schoen, Harald (2006): Beeinflusst Angst politische Einstellungen? Eine Analyse der öffentlichen Meinung während des Golfkriegs 1991. In: Politische Vierteljabresschrift, 47. Jg., Heft 3, 441-464.

Slater, Michael D. (2004). Operationalizing and Analyzing Exposure: The Foundation of Media Effects Research. In: Journalism and Mass Communication Quarterly, 81. Jg., Heft 1, 168-183.

Slater, Michael D. (2007): Reinforcing spirals: The Mutual Influences of Media Selectivity and Media Effects and Their Impact on Individual Behavior and Social Identity. In: Communication Theory, 17. Jg., Heft 2, 281-303.

Thorson, Esther/Christ, William G./Caywood, Clark (1991): Effects of Issue-Image Strategies, Attack and Support Appeals, Music, and Visual Content in Political Commercials. In: Journal of Broadcasting and Electronic Media, 35. Jg., Heft 4, 465-486.

Witte, Erich H. (1992): Das Einstellungskonzept: Alte Themen, neue Klärungsversuche und ein theoretisches Modell. In: Witte, Erich H. (Hrsg.), Einstellung und Verbalten. Braunschweig: Braunschweiger Studien, 35-60.

Zanna, Mark P./Rempel, John K. (1988): Attitudes: A New Look at an Old Concept. In: Bar-Tal, Daniel/Kruglanski, Arie W. (Hrsg.), The Social Psychology of Knowledge. Cambridge: Cambridge University Press, 315-334. 
Anhang 1

Kampagnenmotive der Befürworter der Asylgesetzverschärfung
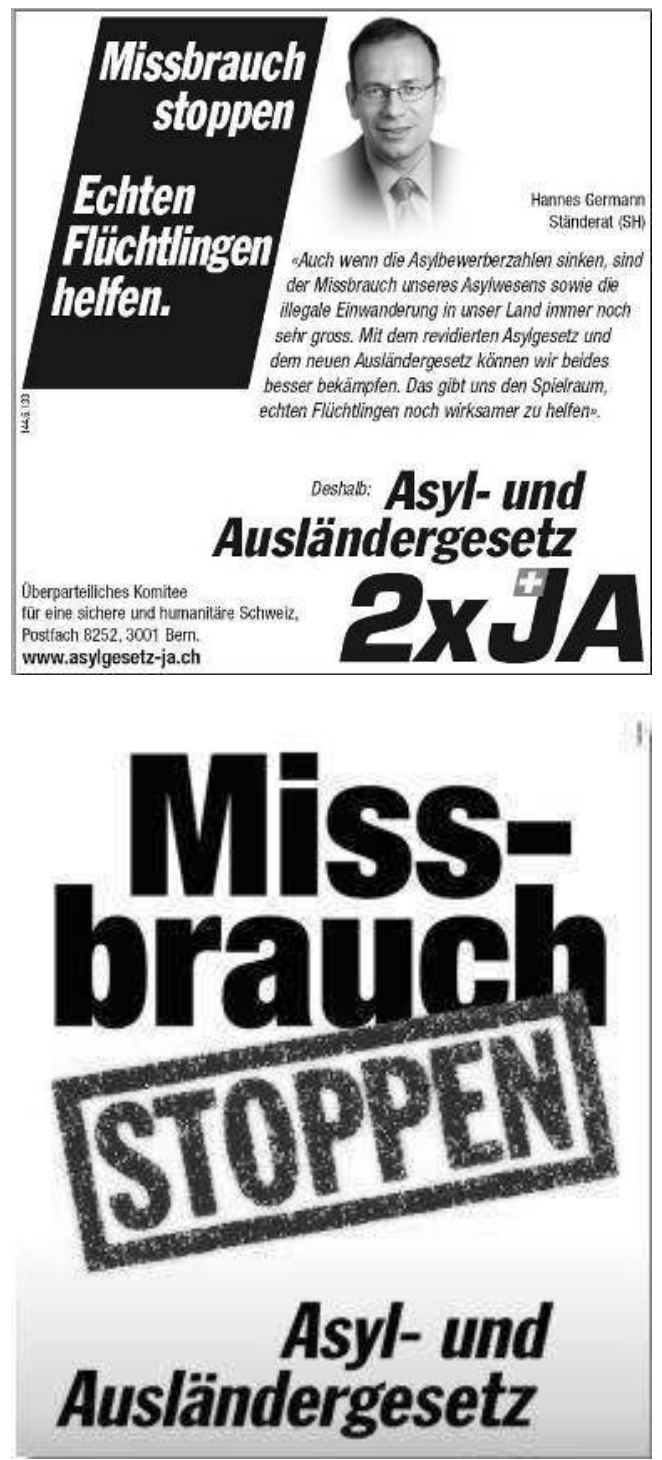
Kampagnenmotive der Gegner der Asylgesetzverschärfung
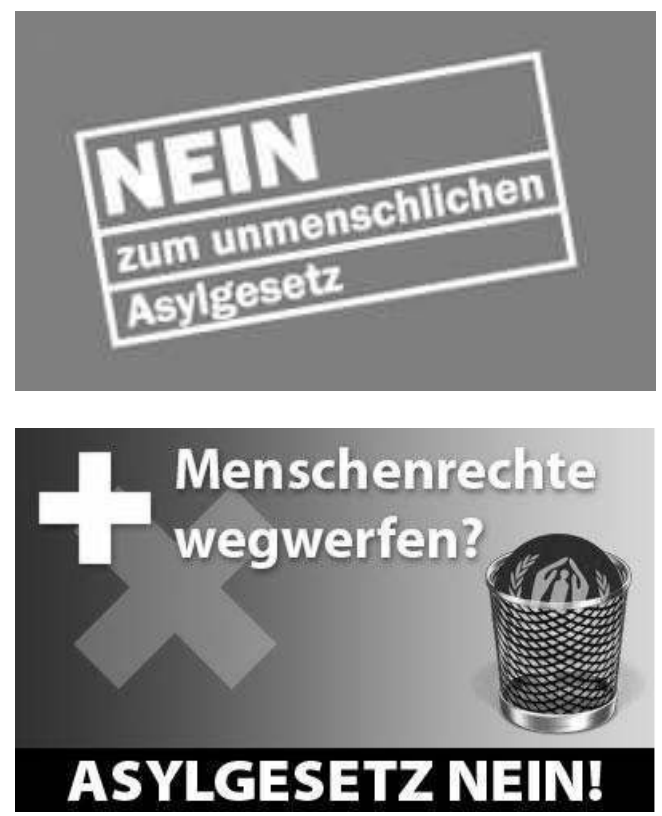


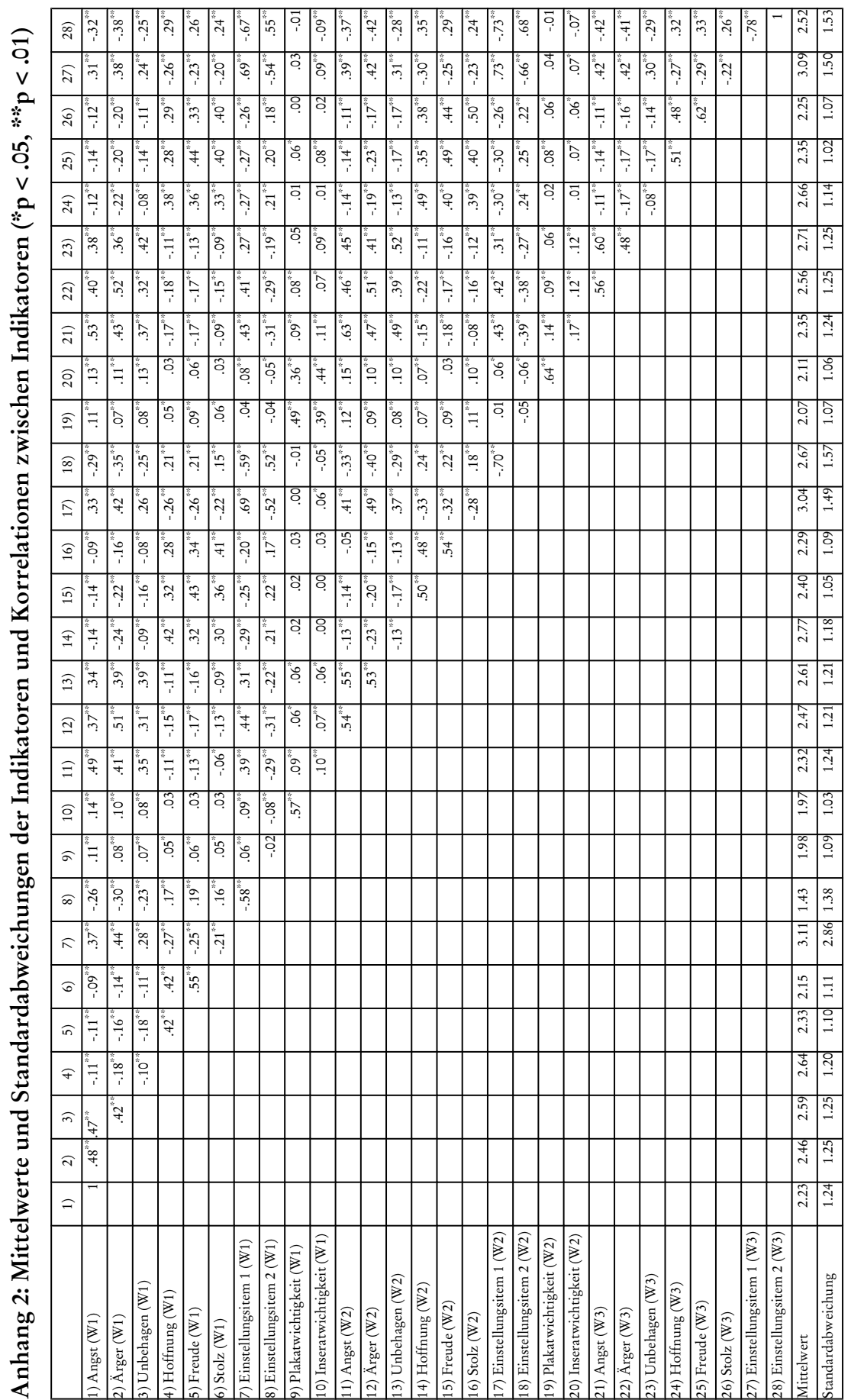

